\title{
FATORES INFLUENTES E EFEITOS DA CONCILIAÇÃO ENTRE O TRABALHO E A FAMÍLIA
}

\author{
Susana Pereira ${ }^{1}$ \\ Célia Ribeiro ${ }^{2}$ \\ Paulo Pereira ${ }^{3}$
}

\begin{abstract}
Resumo: Na sociedade atual, o trabalho e a família constituem o universo estruturante da grande maioria dos atores de uma sociedade. As alterações sociais, nomeadamente o constante reforço das exigências familiares e profissionais, são sem dúvida consequência da temática da conciliação entre estas duas esferas. Torna-se fundamental o conhecimento dos diversos fatores influentes, assim como dos efeitos, no que concerne à conciliação entre o trabalho e a família.
\end{abstract}

Palavras-chave: Conciliação, Trabalho, Família, Fatores influentes, Efeitos

\section{Title: INFLUENTIAL FACTORS AND EFFECTS OF CONCILIATION BETWEEN WORK AND FAMILY}

\begin{abstract}
In today's society, work and family are the structural universe of the vast majority of actors in a society. Social changes, including the constant strengthening of family and work requirements, are undoubtedly a result of the conciliation of these two spheres. The knowledge of the various influencing factors, as well as the effects, is fundamental regarding the conciliation of work and family.
\end{abstract}

Keywords: Conciliation, Work, Family, Influential factors, Effects

\footnotetext{
${ }^{1}$ Mestre em Gestão, Especialização em Gestão de Recursos Humanos. Consultora e formadora na área de segurança, higiene e saúde no trabalho e recursos humanos. Email: scasp@sapo.pt

2 Doutorada em Psicologia, área de especialidade em Psicologia Pedagógica.. Professora Auxiliar da Universidade Católica Portuguesa - Viseu. E-mail: cribeiro@viseu.ucp.pt

${ }^{3}$ Prof. Auxiliar - Departamento de Economia, Gestão e Ciências Sociais da Universidade Católica Portuguesa. E-mail: ppereira@ viseu.ucp.pt
} 


\section{INTRODUÇÃO}

A conciliação entre o trabalho e a família pode variar em função de diversos fatores, entre eles, os fatores sociais tais como o género e o nível socioeconómico, os fatores individuais como a personalidade e a saúde física e mental, e ainda fatores relacionados com a esfera familiar ou profissional, como a composição do agregado familiar, o apoio social e o tipo de emprego (Silva, 2007).

Para uma melhor compreensão da conciliação entre o trabalho e a família, torna-se necessário identificar, por um lado, os fatores determinantes deste tipo de conciliação ao nível de gestão de tempo, envolvimento psicológico e compromisso perante os domínios profissional e familiar; por outro, identificar as consequências em termos de qualidade de vida, bem-estar e satisfação nos papéis profissional e familiar (Pereira, 2012).

Face ao exposto, o presente artigo tem como objetivo abordar os principais fatores influentes e efeitos da conciliação entre o trabalho e a família.

\section{FATORES INFLUENTES DA CONCILIAÇÃO ENTRE O TRABALHO E A FAMÍLIA}

A literatura sobre os fatores influentes da conciliação entre o trabalho e a família tem-se focado fundamentalmente no tempo investido pelo individuo em cada um dos papéis, profissional e familiar, em que está envolvido (Silva, 2007). Grande parte dos efeitos influentes no conflito e no enriquecimento entre o trabalho e a família são reconhecidos devido ao tempo que retiram ou liberam ao individuo. A personalidade do indivíduo é também muito influente nesta conciliação, pois a influência que as características individuais têm na relação que se estabelece entre o papel profissional e familiar é fundamental (Pereira, 2012). Assim, tem-se em consideração os fatores influentes da conciliação entre o trabalho e a família a partir de três níveis, individuo, trabalho e família (Zhang \& Liu, 2011).

\subsection{Variáveis de Natureza Individual}

Os estudos sobre as relações profissionais e familiares têm abordado essencialmente as variáveis situacionais e ambientais. Apesar de 
adaptarem os indivíduos às situações, as características individuais têm sido alvo de poucos estudos (Carlson, 1999; Wayne, Musisca, \& Fleeson, 2004; Zhang \& Liu, 2011).

Dentro das variáveis individuais podemos considerar a idade, o género, as habilitações literárias dos indivíduos e as características de personalidade (Silva, 2007; Zhang \& Liu, 2011).

\subsubsection{Idade}

$\mathrm{O}$ individuo durante a sua vida atravessa vários ciclos, o que the permite acumular ao longo do tempo experiências, competências e conhecimentos que promovam uma melhor assimilação dos papéis profissional e familiar. Assim, é expectável que os indivíduos mais velhos detenham maiores índices de enriquecimento entre o trabalho e a família (Pereira, 2012). A idade parece ser uma variável de estabilização da perceção do conflito entre os dois domínios, que diminui após a meiaidade. Os indivíduos mais novos exibem maiores índices de conflito entre os domínios profissional e familiar (Kmec, 1999), mas segundo um estudo realizado por Grzywacz, Almeida e McDonald (2002), isto só ocorre no caso das mulheres e na direção da família para o trabalho. Simultaneamente, as mulheres mais novas experimentam maiores índices de enriquecimento do domínio profissional para o domínio familiar, enquanto que os homens mais novos experimentam menor enriquecimento do que os homens mais velhos (Grzywacz \& Marks, 2000a).

\subsubsection{Género}

Uma direção da investigação que tem atraído o interesse dos investigadores é o estudo do efeito mediador que o género pode exercer nas vivências profissionais e familiares (Parasuraman \& Greenhaus, 2002; Pereira 2012).

A relevância das diferenças de género vai além das diferenças biológicas entre homens e mulheres, as suas consequências nas famílias de duplo-rendimento determina as expectativas sociais relativas ao desempenho de certos papéis. A teoria dos papéis de género, direciona as opções dos géneros, e atribui aos homens o trabalho como prioridade e às mulheres a família. É expectável assim, que a perceção do conflito, a sobrecarga e o envolvimento com o domínio profissional seja maior nos homens, e o conflito, a sobrecarga familiar e parental e o envolvimento 
com o domínio familiar seja maior nas mulheres (Byron, 2005; Pereira, 2012).

O apoio social na conciliação entre o trabalho e a família será igualmente diferente segundo o género, assim o apoio social alcançado no domínio profissional está relacionado com a conciliação nos homens e o apoio do cônjuge e familiar está mais relacionado com a conciliação nas mulheres (Aryee, Srinivas \& Tan 2005). Porém, Hill (2005) apurou que quando a cultura de uma empresa confere apoio às necessidades familiares, este é um fator mais decisivo para as mulheres do que para os homens, isto deve-se ao facto de os projetos de apoio familiar nos locais de trabalho serem mais dirigidos às mulheres. Esta situação suscita uma questão bastante pertinente, pois esses projetos de apoio familiar também deveriam ser ajustados às necessidades dos homens (Silva, 2007).

Barnett, Marshall e Pleck (1992) contrariam a teoria dos papéis de género, pois em seu estudo constataram que os homens pertencentes a famílias de duplo-rendimento, tanto o papel profissional como o familiar eram determinantes para a sua identidade e bem-estar pessoal. Apurou-se que, nos homens, um menor nível de apoio conjugal e familiar estava relacionado a um maior conflito trabalho-família, enquanto que um menor envolvimento profissional estava relacionado a um maior enriquecimento família-trabalho (Barnett et al., 1992; Grzywacz \& Marks, 2000a).

\subsubsection{Nível Educacional}

O ponto de vista sociocultural sugere que os indivíduos com menores níveis educacionais detêm menores recursos e naturalmente maiores índices de stress e dificuldades em conciliar os domínios profissional e familiar. Contudo, estudos realizados por vários autores sugerem que os indivíduos com cargos profissionais de maior estatuto percecionam maiores níveis de conflito entre o domínio profissional e familiar, apesar de deterem maiores recursos sociais (Grzywac et al., 2002; Marshall \& Barnett, 1993). Também as revisões efetuadas por Byron (2005) e Zhang e Liu (2011) atestam tais estudos.

A revisão de estudos realizada por Jacobs e Gerson (2004) não evidencia nenhuma relação entre o nível educacional das mulheres e a sua perceção do conflito, embora o estudo de Grzywacz e Marks (2000a) atesta que um menor nível educacional está relacionado com um menor índice de enriquecimento nas mulheres. Grzywacz e colaboradores (2002) asseveram que homens e mulheres com menores índices educacionais 
manifestam menores perceções do conflito entre o domínio profissional e familiar.

\subsubsection{Personalidade}

Os traços de personalidade, para Parasuraman e Greenhaus (2002), são formas estáveis e consistentes de agir e pensar que definem a maneira como os indivíduos avaliam os seus ambientes. Na realidade, quando os traços de personalidade intervêm no uso do tempo e na perceção da tensão influenciam a perceção de conflito, enquanto que o efeito desses mesmos traços na mudança de humor, na promoção da autoestima e confiança e na passagem de competências e comportamentos de um domínio para o outro, influenciam a perceção do enriquecimento (Wayne et al., 2004). Estudos revelam que os traços de personalidade pertinentes para a perceção do conflito são, em alguns casos, díspares dos traços pertinentes para a perceção do enriquecimento (Silva, 2007).

A teoria da personalidade mais estudada, o big five, atraiu a atenção nos últimos anos, apesar de a sua pesquisa ser muito escassa (Zhang \& Liu 2011). Esta teoria identifica o traço de neuroticismo que envolve a tensão, a insegurança e a preocupação, estando próximo do conceito de afetividade negativa que revela uma propensão geral de ver o mundo de uma forma negativa. Então, os indivíduos com elevados índices de neuroticismo percecionam as situações como stressantes ou criam situações tendencialmente tensas e interpretam as suas experiências de vida de forma difícil. A tendência destes indivíduos em realçar os aspetos negativos deles próprios e da situação sugere que eles se sentem menos aptos para orientar de forma satisfatória a relação entre o domínio profissional e familiar (Silva, 2007). O neuroticismo e a afetividade negativa estão relacionados assim com a perceção do conflito, mas não com a perceção do enriquecimento (Aryee et al.,2005; Carlson, 1999; Grzywacz \& Marks 2000a; Wayne et al., 2004).

Outras variáveis da personalidade, como a extroversão ou a consciência, parecem exercer um papel na relação entre o trabalho e a família. A extroversão está ligada a indivíduos ativos, assertivos, energéticos e entusiásticos, então, por um lado, os indivíduos com níveis de energia elevados cumprem mais rápida e eficiente as tarefas, e por outro, a propensão de focar os aspetos positivos das situações pode ajudar na perceção destas como menos stressantes. Assim, a extroversão surge associada a maiores índices de enriquecimento (Grzywacz \& Marks 
2000a; Wayne et al., 2004) e a menores índices de conflito (Grzywacz \& Marks, 2000a).

Os indivíduos com elevada consciência tendem a trabalhar afincadamente nos dois domínios de modo atingir os seus objetivos, o que causa o aumento da tensão e conflito, mas estes indivíduos são igualmente eficientes e organizados de modo a diminuir a probabilidade do conflito acontecer. Então, a consciência quando associada à orientação para a tarefa, eficiência e organização gera menores índices de conflito trabalho-família e conflito família-trabalho e maior enriquecimento família-trabalho (Wayne et al., 2004).

A amabilidade é uma variável associada à simpatia, empatia, confiança, e segundo Wayne e colaboradores (2004), parece interagir negativamente com o conflito trabalho-família, e positivamente com o enriquecimento família-trabalho. A variável de abertura à experiência, que inclui a inteligência, imaginação, curiosidade, criatividade e originalidade está relacionada positivamente com o enriquecimento do trabalho para a família e não apresenta qualquer relação com o conflito (Wayne et al., 2004).

O impacto que os traços de personalidade desempenham na perceção do conflito e do enriquecimento atesta a importância destas dimensões. $\mathrm{Na}$ realidade, os traços mais relacionados com a perceção do conflito, como a consciência e o neuroticismo, são os que menos influenciam a perceção do enriquecimento. As investigações sobre a personalidade permitiram ainda averiguar que a extroversão é uma dimensão que influencia as duas direções do enriquecimento (Silva, 2007; Zhang \& Liu, 2011).

\subsection{Variáveis do Domínio Profissional}

O domínio profissional impõe ao individuo uma pluralidade de exigências. $O$ trabalho implica em primeiro lugar um compromisso praticamente total em termos de tempo; segundo, o cumprimento diário das atividades profissionais acarreta física e intelectualmente imposições, e em terceiro, os horários de trabalho, por vezes inadaptáveis às necessidades dos trabalhadores, sobretudo trabalhadores com filhos (Kmec, 1999).

Pela revisão de literatura, acerca dos vários fatores mais relacionados com a perceção do conflito, destaca-se o número de horas e o horário de trabalho, o grau de exigências e pressões no trabalho, o grau de satisfação 
com o desempenho profissional, a qualidade do papel profissional, o desafio profissional e a cultura organizacional (Grzywacz \& Marks, 2000a; Voydanoff, 2002).

\subsubsection{Papel Profissional}

Apesar dos resultados da investigação sobre a temática apresentarem uma grande diversidade, acredita-se que as características do papel profissional tenham influência direta no conflito trabalho-família (Zhang \& Liu, 2011).

As exigências do papel profissional provocam tensão, irritabilidade, ansiedade e fadiga (Silva, 2007). A sobrecarga, que diz respeito à magnitude das exigências profissionais e ao sentimento da pluralidade de tarefas e o curto tempo para as cumprir, influencia a perceção do conflito trabalho-família (Aryee et al., 2005; Greenhaus \& Beutell, 1985; Grzywacz \& Butler, 2005; Grzywacz \& Marks, 2000b; Parasuraman \& Simmers, 2001). Isto acontece pois a sobrecarga aumenta a fadiga e a exaustão que influem negativamente a motivação do individuo para dar resposta às exigências de outros papéis (Aryee et al., 2005; Demerouti, Bakker, \& Schaufeli 2005).

Segundo vários autores (Bedeian, Burke \& Moffett, 1989; Parasuraman \& Simmers, 2001; Wayne, et al., 2004), a sobrecarga, o stress e o conflito no trabalho têm sido relacionados a maiores níveis de conflito entre os domínios, tanto para homens como mulheres. Maiores exigências no trabalho têm um impacto negativo no domínio profissional, no domínio familiar e na relação entre ambos. Todavia, o stress profissional exerce maior efeito na perceção do conflito trabalho-família do que na perceção do conflito família-trabalho (Byron, 2005).

As exigências do domínio profissional e o grau de amplitude das decisões são dois requisitos essenciais para o desenvolvimento de stress ou tensão no papel profissional (Butler, Grzywacz, Bass, \& Linney, 2005). Indivíduos que desempenhem trabalhos com elevadas exigências e com alta liberdade de decisão podem cooperar para o desenvolvimento de novos padrões de comportamento no papel profissional, e também noutros papéis sociais. Assim, os empregos com elevadas exigências e elevada amplitude de decisão promovem maiores índices de enriquecimento trabalho-família (Silva, 2007). Tal situação poderá ser explicada pelo facto do controlo sobre as tarefas implicar flexibilidade quanto à sua concretização e promover um alto sentido de autoestima, 
colaborando assim para o enriquecimento. $\mathrm{O}$ grau de controlo tem sido assim associado a menor conflito trabalho-família (Butler et al., 2005; Grzywacz \& Butler, 2005; Grzywacz \& Marks, 2000b). Enquanto alguns estudos relatam que a um maior nível de competências está associado um menor conflito (Grzywacz \& Butler, 2005), outros não demonstram qualquer relação (Butler et al., 2005).

Constata-se que existe uma variedade de resultados contraditórios quanto às características do papel profissional na perceção do conflito.

\subsubsection{Envolvimento Profissional}

Segundo Carlson e Frone (2003), tanto o envolvimento profissional como o familiar têm exibido resultados inconsistentes na perceção do conflito. Para os autores, entende-se como envolvimento profissional a importância que o domínio profissional tem na autoimagem pessoal e o grau de reconhecimento psicológico com o trabalho. Enquanto que o envolvimento familiar está relacionado com a importância dada ao papel familiar para a autoimagem do indivíduo e ao grau de identificação psicológica com papéis familiares. Se um papel é bastante importante ou central para o autoconceito de um individuo, este produz fortes influências (positivas e negativas) nas outras áreas de vida. $\mathrm{O}$ envolvimento de um individuo com um determinado papel é assim dependente da importância que esse papel tem para a sua autoimagem.

$\mathrm{O}$ envolvimento profissional engloba por um lado um elemento comportamental, que se relaciona com o tempo, a energia investida no papel e com os recursos físicos para ele canalizados, e por outro, um elemento psicológico que implica o investimento de recursos cognitivos e emocionais no desempenho do papel (Carlson \& Frone, 2003). Assim, o envolvimento comportamental e psicológico num dos papéis, profissional ou familiar, dificulta o cumprimento das exigências do outro papel (Greenhaus \& Beutell, 1985).

Verifica-se que o efeito do envolvimento profissional na perceção do enriquecimento não tem sido estudado tão profundamente como o conflito. No entanto, é expectável que o envolvimento com um papel motive os indivíduos a alcançar certos recursos, como competências e apoio, que auxiliem o desempenho do outro papel (Aryee et al., 2005).

\subsubsection{Gestão do Tempo}

No estudo da conciliação entre o trabalho e a família, o número de horas perdidas no trabalho profissional (pago) é considerada a variável 
chave (Barnett, 1998; Greenhaus \& Beutell, 1985). Como o tempo é um recurso limitado, se um individuo gasta mais horas para cumprir as responsabilidades de um papel, resta-lhe menos tempo para cumprir as exigências do outro papel.

Atualmente, os casais de duplo-rendimento despendem um maior número de horas no trabalho pago, porém as investigações que estudam a relação entre o número de horas e o conflito entre o trabalho e a família não são consistentes nas suas conclusões. Se por um lado, uns estudos relatam que a um maior número de horas de trabalho pago está associado maior conflito trabalho-família (Kmec, 1999), outros não demonstram relações consideráveis, e outros estudos ainda referem que os indivíduos que despendem um maior número de horas de trabalho apresentam melhores índices de saúde mental e física, mas uma maior insatisfação do/a companheiro/a (Barnett, 1998).

A inconsistência destas conclusões poderá ser justificada, se considerarmos que os empregos nos quais os indivíduos despendem um maior número de horas de trabalho são aqueles que incluem bons salários, mais benefícios, atividades mais complexas e desafiantes, como os advogados, do que empregos com horários mais reduzidos (Silva, 2007). Para estimar o impacto do número de horas de trabalho na perceção da interferência entre o domínio profissional e familiar, todos estes fatores devem ser considerados. Na realidade, os indivíduos com estatutos profissionais mais elevados percecionam menores índices de conflito do que os indivíduos de estatutos mais baixos (Kmec,1999). O número de horas despendido no trabalho não parece ter qualquer relação com a perceção do enriquecimento entre o domínio profissional e familiar (Voydanoff, 2004).

O tempo despendido no local de trabalho e a sua interação com o bem-estar do indivíduo e a sua perceção do conflito trabalho-família, não deve ser encarado como uma relação direta. Deve-se analisar o impacto que um maior número de horas de trabalho pago poderá ter no conflito, sob determinadas condições e para alguns grupos de trabalhadores. Também é necessário destrinçar o tempo de trabalho de outros fatores que lhe podem estar ou não associados, como a flexibilidade, o controlo do horário de trabalho, a autonomia ou um ambiente de trabalho apoiante (Silva, 2007). Em suma, o número de horas de trabalho pago é afetado pela natureza e estrutura do trabalho, principalmente pelo grau de controlo que o individuo tem sobre as atividades. 


\subsubsection{Flexibilidade e Apoio Profissional}

A flexibilidade profissional, através de maior autonomia, maior controlo sobre os horários de trabalho e maior apoio organizacional diminui a perceção do conflito entre os dois domínios (Byron, 2005; Stevens Minnotte \& Kiger 2004). Porém, na revisão de estudos de Byron (2005), esta relação aparenta ser mais forte no sentido trabalho-família. É de realçar que a autonomia e a flexibilidade de horários estão relacionadas, mas são conceptualmente distintos (Stevens et al., 2004). A existência de horários de trabalho não estandardizados está associada a maiores índices de conflito, mas se o individuo tiver controlo sobre estes horários a perceção do conflito será menor (Silva, 2007).

$\mathrm{O}$ apoio profissional através de medidas de apoio à família está associado a menores índices de conflito trabalho-família, maiores índices de enriquecimento trabalho-família e a maiores índices de satisfação profissional e familiar (Hill, 2005). Porém, apesar da relação entre a existência de benefícios familiares no local de trabalho e maior compromisso organizacional e produtividade (Hill, 2005), verifica-se que a existência de práticas no local de trabalho que garantam flexibilidade e controlo dos papéis profissionais e familiares não garante que os indivíduos sejam mais produtivos e leais que outros que não dispõem de tais práticas (Barnett,1998). A existência de apoio profissional pode moderar as consequências do stress, sendo assim um amortecedor dos efeitos do conflito, e ao mesmo tempo promotor dos papéis profissionais e familiares, na medida em que promove a flexibilidade. No lado oposto, a pressão profissional está relacionada com a perceção do conflito (Hill, 2005; Mauno Kinnunen \& Ruokolainen, 2006).

De referir, que mais uma vez, são poucos os estudos que procuram analisar a relação das variáveis do domínio profissional e o processo de enriquecimento.

\subsection{Variáveis do Domínio Familiar}

As variáveis do domínio familiar influenciam a conciliação entre o trabalho e a família através das situações vivenciadas pelo individuo. A pluralidade das responsabilidades do individuo está relacionada com o estatuto profissional do cônjuge, com a presença de filhos, com o apoio familiar recebido e pela divisão de tarefas domésticas (Kmec, 1999).

As variáveis mais estudadas deste domínio incluem as variáveis da tipologia familiar, das exigências do papel, apoio recebido, envolvimento 
e satisfação com o papel marital e parental, número de filhos e dinâmica familiar (Silva, 2007; Zhang \& Liu, 2011).

\subsubsection{Tipologia Familiar}

O número de papéis que um individuo desempenha aumenta com o casamento, através do aumento da família, do número de eventos familiares e também pelo número de familiares que precisem de apoio. Porém, a investigação demonstra que a relação entre este fator e a conciliação entre o trabalho e a família depende do cônjuge estar ou não empregado (Hill, 2005; Kmec, 1999). Com a entrada massiva das mulheres no mercado de trabalho, aumentam os estudos das dinâmicas das famílias de duplo-rendimento, pois ambos elementos do casal desempenham uma atividade profissional. Parte-se da premissa que este tipo de famílias estará sujeita a maiores interferências entre os domínios, apresentando assim maiores níveis de conflito (Grzywacz \& Marks, 2000a). Na realidade, os indivíduos que não são casados manifestam um menor conflito trabalho-família mas também um menor enriquecimento família-trabalho (Grzywacz \& Marks, 2000a).

\subsubsection{Estatuto Parental}

$\mathrm{Na}$ estrutura familiar, a existência de filhos em casa é dos fatores que exerce uma influência mais determinante no aumento da perceção do conflito, tanto maior quanto mais pequenos e dependentes forem os filhos e sobretudo para as mulheres (Grzywacz \& Marks, 2000a; Jacobs \& Gerson, 2004). De realçar que a existência de filhos está mais relacionada com a perceção do conflito família-trabalho do que com o conflito trabalho-família (Byron, 2005).

$\mathrm{Na}$ análise das relações entre o domínio profissional e familiar, deve ser tido em consideração o período de vida em que a família se encontra, principalmente quanto à existência de filhos. A existência de filhos contribui assim para uma maior perceção de stress e maior dificuldade na gestão dos papéis profissionais e familiares, mas contribui igualmente para a perceção do enriquecimento que pode influenciar o desempenho do indivíduo nos diferentes papéis (Grzywacz \& Marks, 2000a; Hill, 2005). 


\subsubsection{Dinâmica Familiar}

As variáveis da dinâmica familiar como o apoio, a coesão e o grau de conflito familiar estão associados ao conflito entre os domínios familiar e profissional.

As emoções vivenciadas por um membro da família poderão influenciar o estado emocional do parceiro (Barnett, 1998). Assim, o aumento da intensidade do conflito de papel e da tensão emocional de um dos elementos promove o aumento da intensidade do conflito e da tensão do parceiro, que por sua vez aumenta a perceção de conflito e de tensão do primeiro elemento (Silva, 2007). Porém, os efeitos negativos associados ao stress dos cônjuges podem ser suavizados pelas esposas, quando estas os dispensam da execução das tarefas familiares (Barnett, 1998). Os casais que têm maior envolvimento profissional tendem a vivenciar menor índice de conflito (Beutell \& Greenhaus, 1982). Tal facto propõe que quando ambos os membros estão bastante envolvidos no papel profissional, os parceiros compreendem melhor as necessidades e ambições do cônjuge, adaptando as suas expectativas em relação ao envolvimento do outro nos papéis familiares e desenvolvendo-se assim uma adaptação mútua.

$\mathrm{O}$ apoio social, em especifico o apoio do cônjuge, é outro aspeto essencial da dinâmica familiar, importante na relação entre os papéis profissional e familiar (Parasuraman \& Greenhaus, 2002). O apoio do cônjuge, com o objetivo de ajudar o parceiro e promover o seu bem-estar, tem sido relacionado com menores índices de conflito trabalho-família e maiores índices de enriquecimento entre os dois domínios (Aryee et al., 2005; Grzywacz \& Marks, 2000a).

\subsubsection{Exigências Familiares}

A pressão e o stress familiar interferem negativamente no desempenho do papel profissional (Carlson, 1999; Higgins \& Duxbury, 1992). Segundo a revisão de Byron (2005), maiores índices de stress familiar estão relacionados com uma maior perceção do conflito famíliatrabalho e menor stress familiar, segundo alguns estudos, está associado a maior enriquecimento família-trabalho (Grzywacz \& Marks, 2000a).

Após várias referências sobre o uso do tempo, verifica-se que a um maior número de horas gasto em atividades familiares, como tarefas domésticas e cuidado dos filhos, está associado maiores perceções de conflito (Greenhaus \& Beutell, 1985), particularmente com maiores níveis de conflito família-trabalho (Byron, 2005). Hill (2005) relatou que 
o número de horas gasto em atividades domésticas não tinha qualquer relação com o conflito ou enriquecimento trabalho-família, apesar das tarefas de cuidado dos filhos estarem associadas a maiores índices de enriquecimento trabalho-família, bem como a satisfação com a vida, pois facilita a gestão do stress individual.

Tendo em conta que as mulheres são as responsáveis pelo cumprimento da maioria das tarefas domésticas e cuidado dos filhos, mesmo quando realizam trabalho pago a tempo inteiro, o papel do cônjuge é relevante na perceção do conflito entre os domínios profissional e familiar. As investigações sugerem que a insatisfação na divisão de tarefas domésticas está relacionada com o maior conflito conjugal, enquanto que uma maior partilha de tarefas está associada a menores índices de conflito profissional e familiar para as mulheres, embora existam estudos que tenham chegado a conclusões opostas. Baruch, Biener e Barnett (1986) verificaram que a participação dos homens em tarefas tradicionalmente femininas estava relacionada a maiores níveis de conflito nas mulheres. Uma possível explicação poderá estar no facto de as mulheres consideravam que deveriam gerir todas as tarefas profissionais e familiares e percecionavam a participação dos seus cônjuges nas tarefas de cuidado dos filhos como uma incapacidade sua em cumprir com o seu papel de mãe.

\section{EFEITOS DA CONCILIAÇÃO ENTRE O PAPEL PROFISSIONAL E FAMILIAR}

O conflito entre os domínios profissional e familiar tem sido identificado como gerador de efeitos negativos a nível pessoal, que vão desde a menor saúde mental e física, stress, ansiedade, irritabilidade, insatisfação, agressividade, perda de apetite, fadiga, tensão, diminuição do bem-estar psicológico, depressão, diminuição do desempenho dos papéis parentais (Frone, Russell \& Cooper, 1992a, 1992b), menor satisfação com a vida (Hill, 2005), maior probabilidade de desenvolver estilos de vida negativos como o consumo de substâncias, álcool e tabaco (Frone et al., 1992a) e Burnout. O Burnout é uma síndrome da exaustão emocional, física e mental do individuo, de despersonalização e de uma redução da realização pessoal (Maslach \& Jackson, 1984). A exaustão emocional refere-se a um esgotamento dos recursos emocionais (Maslach \& Jackson, 1984) e envolve sentimentos de incapacidade, inutilidade, 
choro incontrolável, podendo levar no extremo a uma quebra emocional ou pensamentos suicidas (Pereira, 2009). A exaustão física é descrita por baixa energia, fadiga crónica, fraqueza e queixas de frequentes dores de cabeça, náuseas e tensão muscular (Pereira, 2009). E, por último a exaustão psicológica é caracterizada pelo desenvolvimento de atitudes negativas em relação a si próprio, ao trabalho e à vida (Pereira, 2009). De facto, os profissionais, vítimas de Burnout, sentem-se inadequados, inferiores e incompetentes (Pereira, 2009). A nível das relações, o conflito entre o domínio profissional e pessoal, poderá levar a um aumento dos conflitos interpessoais, divórcio, entre outros (Duxbury \& Higgins, 1991).

As revisões elaboradas por diversos autores verificaram correlações negativas entre o conflito e a satisfação com a vida (Allen, Herst \& Bruck, 2000; Netemeyer Boles \& McMurrian, 1996). Porém, alguns investigadores consideram que esta relação poderá ser mediada pelo nível de satisfação profissional e familiar (Aryee et al., 2005; Bedeian et al., 1989). Desta forma, o conflito causaria menores índices de satisfação profissional e familiar que influenciariam de forma negativa o índice de satisfação global com a vida. O conflito trabalho-família poderá ser interpretado como um indicativo da inaptidão do indivíduo na gestão das tarefas familiares, quando este julga que possui menor controlo sobre as interferências do papel profissional sobre o papel familiar (Silva, 2007).

Porém, há estudos que sugerem que o desempenho concomitante, tanto em homens como em mulheres de vários papéis, como o papel de cônjuge, de pais e de trabalhadores, conduzem a elevados índices de bemestar psicológico, comparativamente com aqueles que desempenham um menor número de papéis (Voydanoff, 2002). Isto ocorre porque o cumprimento de qualquer um desses papéis gera por um lado recompensas externas, que podem ser monetárias ou até mesmo o reconhecimento dos outros, e por outro, recompensas internas, como o sentimento de satisfação pessoal ou de autorrealização (Silva, 2007). Estas recompensas poderão estar relacionadas a emoções, a estados de humor positivos como a satisfação, a alegria e o orgulho, enquanto que a falta de um ou outro desses papéis poderá originar frustração, insatisfação, deceção e tristeza (Silva, 2007).

$\mathrm{Na}$ direção das consequências do conflito de papéis, é deveras importante distinguir dois tipos. Quando o conflito ocorre ao nível do trabalho e se repercute na vida familiar, destacam-se as consequências ao nível do indivíduo (stress, ansiedade, irritabilidade, insatisfação, etc.) e ao 
nível da dinâmica familiar (insatisfação geral com a família e com a relação conjugal, fraca participação nas atividades familiares, ausência em momentos importantes da vida familiar e dificuldades de interação com os filhos) (Frone, 2000). Quando o conflito se manifesta da família para o trabalho, destacam-se consequências como baixo desempenho profissional, insatisfação profissional, elevado absentismo, diminuição de produtividade, baixa moral, perda de talentos e mudanças frequentes de atividade profissional, bem como problemas de saúde física e mental (Carlson Kacmar \& Williams., 2000; Carlson \& Frone, 2003; Duxbury \& Higgins,1991; Frone, 2000). Estas consequências são cumulativas quando o conflito é bidirecional, ou seja, conflito trabalho-família e conflito família-trabalho, ou quando o sujeito exibe determinadas características de personalidade, como a introversão, elevado neuroticismo e baixa autoestima (Carlson \& Frone, 2003; Frone, 2000).

Quando as exigências e responsabilidades associadas a um dos papéis interferem com frequência no desempenho do outro papel, a qualidade de vida associada a esse segundo papel é influenciada (Frone et al.,1992a). Deste modo, a perceção de conflito do domínio familiar para o domínio profissional está positivamente relacionada com stress, insatisfação e menor desempenho profissional enquanto que a perceção de conflito do domínio profissional para o domínio familiar está relacionada com stress, insatisfação e menor desempenho familiar.

Em síntese, as consequências do conflito são abundantes, além de muito estudadas e documentadas em relação à insatisfação profissional, diminuição da produtividade e absentismo, enquanto que as suposições do enriquecimento são pouco claras, devido ao baixo número de investigações efetuadas para esta relação. Porém, partindo do pressuposto que a gestão de múltiplos papéis promove um melhor desempenho individual, então maiores índices de enriquecimento estarão relacionados a maior satisfação profissional e pessoal, a maior envolvimento organizacional e consequente maior produtividade (Aryee et al., 2005).

\section{REFLEXÕES CONCLUSIVAS}

A conciliação entre o domínio profissional e familiar é bidirecional e cada direção possui fatores influentes e efeitos distintos. De uma maneira geral, os processos que se desenvolvem no domínio profissional estão mais relacionados à conciliação no sentido do trabalho para a família, e os 
processos desenvolvidos no seio da família estão mais relacionados com a conciliação da família para o trabalho (Byron, 2005). As investigações também distinguem o tipo de fatores influentes habitualmente associados ao enriquecimento e ao conflito. Assim, o enriquecimento parece estar mais associado a recursos, enquanto que o conflito está mais associado a exigências e stress (Voydanoff, 2004, 2005). Apesar do conflito estar relacionado com a pressão profissional e familiar, o apoio do cônjuge parece estar somente associado ao conflito família-trabalho. $\mathrm{O}$ mesmo se verifica em relação ao enriquecimento. Se, por um lado, as variáveis como grau de autonomia e de controlo estão associadas ao enriquecimento, o apoio do cônjuge está associado ao enriquecimento família-trabalho, mas não trabalho-família.

Em relação aos efeitos negativos da perceção do conflito, torna-se claro, pelos estudos referidos, que o conflito manifesta sérios efeitos ao nível individual, familiar e organizacional. O conflito suscita custos sociais ao nível da vida profissional e familiar do indivíduo, ao nível do seu bem-estar e saúde em geral.

Da revisão da literatura efetuada constatou-se que as características individuais têm um papel predominante no uso dos recursos. Apesar de os indivíduos suportarem um mesmo conjunto de stressores, a maneira como lidam e gerem esta tensão é influenciada por fatores individuais. Logo, o índice de conflito ou, por outro lado, do enriquecimento resulta não só das características profissionais e familiares mas também das características individuais.

\section{BIBLIOGRAFIA}

Allen, T. D., Herst, D. E., \& Bruck, C. S. (2000). Consequences associated with work to- family conflict: a review and agenda for future research. Journal of Occupational Health Psychology, 5, 2, 278-308.

Aryee, S, Srinivas, E. \& Tan, H. (2005). Rhythms of life: antecedents and outcomes of work-family balance in employed parents. Journal of Applied Psychology, 90 (1) 132-146.

Barnett, R. (1998). Toward a review and reconceptualization of the work/family literature. Genetic, Social \& General Psychology Monographs, 124 (2), 125-153. 
Barnett, R., Marshall, N., \& Pleck, J. (1992). Men's multiple roles and their relationship to men's psychological distress. Journal of Marriage and the Family, 54, 358-367.

Baruch, G., Biener, L. \& Barnett, R. (1986). Women and gender in research on work and family stress. American Psychologist, 42 (2), 130-136.

Byron, K. (2005). A meta-analytic review of work-family conflict and its antecedents. Journal of Vocational Behavior, 67, 169-198.

Bedeian, A.G., Burke, B.G., \& Moffett, R.G. (1989). Outcomes of workfamily conflict Among married male and female professionals. Journal of Management, 14, (3), 475-491.

Beutell, N. \& Greenhaus, J. (1982). Interrole conflict among married women: the influence of husband and wife characteristics on conflict and coping behaviour. Journal of Vocational Behaviour, 21, 99-110.

Butler, A, Grzywacz, J., Bass, B., \& Linney, K. (2005). Extending the demands-control model: A daily diary study of job characteristics, work-family conflict and work-family facilitation. Journal of Occupational and Organizational Psychology, 78, 155 - 169.

Carlson, D. (1999). Personality and Role Variables as predictors of three forms of work-family conflict. Journal of Vocational Behaviour, 55, 236-253.

Carlson, D. \& Frone, M. (2003). Relation of behavioural and psychological involvement to a new four-factor conceptualization of work-family interference. Journal of Business and Psychology, 17 (4), 515-535.

Carlson, D. S., Kacmar, K. M., \& Williams, L. J. (2000). Construction and initial validation of a multidimensional measure of work-family conflict. Journal of Vocational Behavior, 56, 249-276.

Demerouti, E, Bakker, A. \& Schaufeli, W. (2005). Spillover and crossover of exhaustion and life satisfaction among dual-earner parents. Journal of Vocational Behaviour 67, 266-289.

Duxbury, L.E. \& Higgins, L.A. (1991). Gender differences in work/family conflict. Journal of Applied Psychology, 78, (1), 60-73.

Frone, M., Russell, M., \& Cooper, M. (1992a). Antecedents and outcomes of work-family conflict: testing a model of the work-family interface. Journal of Applied Psychology, 77 (1), 65-78. 
Frone, M., Russell, M., \& Cooper, M. (1992b). Prevalence of workfamily conflict: are work and family boundaries asymmetrically permeable? Journal of Organizational Behavior, 13, 723-729.

Frone, M (2000). Work-family conflict and employee psychiatric disorders: the national comorbidity survey. Journal of Applied Psychology, 85 (6), 888-895.

Greenhaus, J.H. \& Beutell, N.J. (1985). Sources of conflict between work and family roles. Academy of Management Review, 10 (1), 76-88.

Grzywacz, J., Almeida, D. \& McDonald, D. (2002). Work-family spillover and daily reports of work and family stress in the adult labor force. Family Relations, 51, 28-36.

Grzywacz, J. \& Butler, A. (2005). The impact of job characteristics on work-to-family facilitation: testing a theory and distinguishing a construct. Journal of Occupational Health Psychology, 10 (2), 97-109.

Grzywacz, J.G., \& Marks, N.F. (2000a). Reconceptualizing the workfamily interface: An ecological perspective on the correlates of positive and negative spillover between work and family. Journal of Occupational Health Psychology, 5, 111-126.

Grzywacz, J. \& Marks, N. (2000b). Family, work, work-family spillover and problem drinking during midlife. Journal of Marriage and the Family, 62, 336-348.

Higgins, C \& Duxbury, L. (1992).Work-family conflict: A comparison of dual-career and traditional career men. Journal of Organizational Behaviour, 13, 389-411.

Hill, J. (2005). Work-Family facilitation and conflict, working fathers and mothers, work-family stressors and support. Journal of Family Issues, 26 (6), 793-819.

Jacobs, J. A. \& Gerson, K. (2004). The time divide: work, family and gender inequality. Harvard University Press: Cambridge.

Kmec, J. (1999). Multiple aspects of work-family conflict. Sociological Focus, 32 (3), 265-285.

Marshall, N., \& Barnett, R. (1993). Work-family strains and gains among two-earner couples. Journal of Community Psychology, 21, 64-78.

Maslach, C \& Jackson, S.E. (1984). Burnout in organizational settings. Applied Social Psychology Annual, 5, 133-153.

Mauno, S., Kinnunen, U., \& Ruokolainen, M. (2006). Exploring work and organization based resources as moderators between work-family conflict, well-being and job attitudes. Work \& Stress, 20, 3, 210-233. 
Netemeyer, R. G., Boles, J. S., \& McMurrian, R. (1996). Development and validation of work-family conflict and family-work conflict scales. Journal of Applied Psychology, 81, 400-410.

Parasuraman, S. \& Greenhaus, J. (2002). Toward reducing some critical gaps in work-family research. Human Resource Management Review, 12, 299-312.

Parasuraman, S., \& Simmers, C. A. (2001). Type of employment, workfamily conflict and well-being. A comparative study. Journal of Organizational Behavior, 22, 551- 568.

Pereira, A. (2009). Burnout e o Conflito Trabalho-Família / FamíliaTrabalho em Profissionais de Enfermagem. Dissertação de Mestrado Integrado em Psicologia. Faculdade de Psicologia e Ciências da Educação. Universidade do Porto. Porto.

Pereira, S. (2012). O equilíbrio trabalho/família nos advogados: Diferenças de género. Dissertação de Mestrado em Gestão: Especialização em Gestão de Recursos Humanos. Centro regional de Viseu da Universidade Católica Portuguesa. Viseu.

Silva, M. (2007). Vida Profissional e Familiar: Padrões de Conflito e Facilitação na Gestão de Múltiplos Papéis. Dissertação de Mestrado em Psicologia. F.P.C.E.U.P. Porto.

Stevens, D. P., Minnotte, K. L., \& Kiger, G. (2004). Differences in workto-family and family-to-work spillover among professional and nonoprofessional workers. Sociological Spectrum, 24, 535-551.

Voydanoff, P. (2002). Linkages between the work family interface and work, family and individual outcomes: an integrative model. Journal of Family Issues, 23, (1), 138-164.

Voydanoff, P. (2004). Implications of work and community demands and resources for work-tofamily conflict and facilitation. Journal of Occupational Health Psychology, 9 (4), 275-285

Voydanoff, P. (2005). The differential salience of family and community demands and resources for family to work conflict and facilitation. Journal of Family and Economic Issues, 26 (3), 395-417

Wayne, J.H., Musisca, N., \& Fleeson, W. (2004). Considering the role of personality in the work-family experience: Relationships of the Big Five to work-family conflict and enrichment. Journal of Vocational Behavior, 64, 108-130. 
Zhang, J. \& Liu, Y. (2011). Antecedentes of work-family conflit: Review and Prospect. International Journal of Business and Management, 6, 1, 89-103. 